RECEIVED 2016 SEPTEMBER 17; ACCEPTED 2016 OCTOBER 13

Preprint typeset using LTEX style emulateapj v. 5/2/11

\title{
ON THE RADIO DICHOTOMY OF ACTIVE GALACTIC NUCLEI
}

\author{
XINWU CAO ${ }^{1,2}$ \\ Received 2016 September 17; accepted 2016 October 13
}

\begin{abstract}
It is still a mystery why only a small fraction of active galactic nuclei (AGNs) contain relativistic jets. A strong magnetic field is a necessary ingredient for jet formation, however, the advection of the external field in a geometrically thin disk is inefficient. Gas with a small angular velocity may fall from the Bondi radius $R_{\mathrm{B}}$ nearly freely to the circularization radius $R_{\mathrm{c}}$, and a thin accretion disk is formed within $R_{\mathrm{c}}$. We suggest that the external magnetic field is substantially enhanced in this region, and the magnetic field at $R_{\mathrm{c}}$ can be sufficiently strong to drive outflows from the disk if the angular velocity of the gas is low at $R_{\mathrm{B}}$. The magnetic field is efficiently dragged in the disk, because most angular momentum of the disk is removed by the outflows that lead to a significantly high radial velocity. The strong magnetic field formed in this way may accelerate jets in the region near the black hole, either by the Blandford-Payne or/and Blandford-Znajek mechanisms. We suggest that the radio dichotomy of AGNs predominantly originates from the angular velocity of the circumnuclear gas. An AGN will appear as a radio-loud (RL) one if the angular velocity of the circumnuclear gas is lower than a critical value at the Bondi radius, otherwise, it will appear as a radio-quiet (RQ) AGN. This is supported by the observations that RL nuclei are invariably hosted by core galaxies. Our model suggests that the mass growth of the black holes in RL quasars is much faster than that in RQ quasars with the same luminosity, which is consistent with the fact that the massive black holes in RL quasars are systematically a few times heavier than those in their RQ counterparts.
\end{abstract}

Subject headings: accretion, accretion disks, black hole physics, magnetic fields, galaxies: active, galaxies: jets

\section{INTRODUCTION}

Active galactic nuclei (AGNs) can be divided into two categories, i.e., radio-loud (RL) and radio-quiet (RQ) AGNs, according to their ratios of the radio emission to optical emission. Radio emission from RL AGNs is predominantly from the jets, and it is still a mystery why only a small fraction (about one tenth) of AGNs exhibit relativistic jets, while their appearance is quite similar to the RQ counterparts in almost all wavebands except radio wavebands (Kellermann et al. 1989; Xu et al. 1999; Cirasuolo et al. 2003; Baloković et al. 2012).

The Blandford-Znajek (BZ) and Blandford-Payne (BP) mechanisms are the most favored models of jet formation in AGNs (Blandford \& Znajek 1977; Blandford \& Payne 1982). The power of jets is extracted from the accretion disk or the rotating black hole by the large scale magnetic field. In the BZ mechanism, energy and angular momentum are extracted from a rotating black hole by open magnetic field lines, while the magnetic field threading the disk accelerates a small fraction of the gas in the disk to form the jets. Rapidly rotating black holes are required in RL AGNs if the BZ mechanism is responsible for the jet formation, and therefore the black hole spin is regarded as an intrinsic difference between RL and RQ AGNs (Wilson \& Colbert 1995; Ye \& Wang 2005; Sikora et al. 2005, 2007, 2008; Tchekhovskoy et al. 2010). However, it is still in debate the relative importance of the BP and BZ mechanisms (Livio et al. 1999; Cao 2002, 2003; Fender et al. 2010; Tchekhovskoy et al. 2011; Narayan \& McClintock 2012). Although we do not know which mechanism is dominant in the jet formation of AGNs,

\footnotetext{
${ }^{1}$ SHAO-XMU Joint Center for Astrophysics, Shanghai Astronomical Observatory, Chinese Academy of Sciences, 80 Nandan Road, Shanghai, 200030, China; cxw@shao.ac.cn

${ }^{2}$ Key Laboratory of Radio Astronomy, Chinese Academy of Sciences, 210008 Nanjing, China
}

a strong large-scale magnetic field near the black hole is necessary for the jets in RL AGNs either with the BP or BZ mechanisms. It is reasonable to consider the strong magnetic field as a crucial factor causing the radio dichotomy in AGNs (Sikora \& Begelman 2013), though the black hole spin may also play an important role in the BZ scenario. However, the numerical simulations show that almost all massive black holes will soon be spun up to rapidly spinning holes by accreting the gas in the disks (Volonteri et al. 2007), though this may be alleviated if the chaotic accretion is assumed in AGNs (King et al. 2008; Li et al. 2012; Volonteri et al. 2013). This may imply that the radio dichotomy is not solely caused by the black hole spin, instead, the radio dichotomy of AGNs may probably originate from the strong magnetic field near the black hole, i.e., an AGN appears as an RL AGN only if a strong magnetic field is present to drive relativistic jets from the region near a black hole.

It is still unclear how the strong large scale magnetic field is formed in the accretion disk. The external weak largescale poloidal field (e.g., the field threading the interstellar medium) is suggested to be dragged inward by the accretion plasma, which is balanced by the magnetic diffusion in the disk for a steady field case (Bisnovatyi-Kogan \& Ruzmaikin 1974, 1976; van Ballegooijen 1989; Lubow et al. 1994; Ogilvie \& Livio 2001). This means that the configuration of the magnetic field dragged by the disk is predominantly determined by the magnetic diffusivity and the radial velocity of gas in the disk. In a conventional turbulent accretion disk, its radial velocity is mainly regulated by the kinematic viscosity $\nu$, and the advection of the field in the disk is sensitive to the magnetic Prandtl number $P_{\mathrm{m}}=\eta / \nu$, where $\eta$ is the magnetic diffusivity. It was suggested that the magnetic Prandtl number should be around unity, either based on the simple estimate of the order of magnitude (Parker 1979) or the numerical simulations (e.g., Yousef et al. 2003; Lesur \& Longaretti 
2009; Fromang \& Stone 2009; Guan \& Gammie 2009). It was found that the field can hardly be dragged inward by a thin disk $(H / R \ll 1)$ because of its small radial velocity. The magnetic diffusion timescale is about the same as the viscous timescale in a steady thin disk, and the field in the inner region of the disk is not much stronger than the external weak field (Lubow et al. 1994). In order to solve this problem, a few mechanisms were suggested to alleviate the difficulty of field advection in the thin disks (Spruit \& Uzdensky 2005; Lovelace et al. 2009; Guilet \& Ogilvie 2012, 2013; Cao \& Spruit 2013). Cao \& Spruit (2013) suggested that the most angular momentum of the gas in the thin disk can be removed by the magnetically driven outflows, and the radial velocity of the disk is significantly increased. In this case, the external field can be advected efficiently by the disk with magnetic outflows.

The jets are driven by the strong magnetic field in the region near the black hole, either by the BP or/and BZ mechanisms. On the assumption that an AGN will appear as an RL AGN only if a strong magnetic field is formed near the black hole, we develop a model for the origin of the radio dichotomy of AGNs. The model is described in Section 2, and we put the results and discussion in Sections 3 and 4. The final section contains a brief summary.

\section{MODEL}

The gas falls almost freely toward the black hole, if the angular momentum of the gas is significantly lower than the Keplerian value at the Bondi radius. The angular momentum of the gas is roughly conserved until it approaches the circularization radius (e.g., Melia et al. 2001, but also see $\mathrm{Bu} \&$ Yuan 2014). The external weak magnetic field is dragged in by the gas in the region between the Bondi radius and the circularization radius, due to the field flux freezing, and the field threading the gas is substantially enhanced at the circularization radius. An optically thick, geometrically thin accretion disk is formed with the circularization radius, if the gas is supplied at an appropriate rate. The angular momentum of the gas is removed by the turbulence in the accretion disk.

An effective magnetic diffusivity, corresponding to a magnetic Prandtl number of the order of unity, is caused by the turbulence in the disk. The magnetic field advection in a thin accretion disk is quite inefficient due to magnetic diffusion in such a turbulent disk, because the radial velocity of a thin disk is low. It was suggested that its radial velocity is significantly increased due to the presence of the outflows, if the angular momentum of the disk is removed predominantly by the magnetically driven outflows (see Cao \& Spruit 2013, for the details). The field in such a disk with outflows is therefore efficiently advected toward the black hole. The strong magnetic field formed in this way may accelerate relativistic jets in the inner region of the disk near the black hole, either by the BP or/and BZ mechanisms. The object containing such an accretion disk with magnetic outflows may appear as an RL AGN, otherwise, it may be an RQ AGN.

\subsection{Accretion of the gas in the circumnuclear region of the galaxy}

The Bondi radius and the Bondi accretion rate can be calculated by the properties of the circumnuclear gas,

$$
R_{\mathrm{B}}=\frac{2 G M_{\mathrm{bh}}}{c_{\mathrm{s}}^{2}},
$$

and

$$
\dot{M}=4 \pi \lambda\left(G M_{\mathrm{bh}}\right)^{2} c_{\mathrm{s}}^{-3} \rho,
$$

where the sound speed $c_{\mathrm{s}}=\left(\gamma k T / \bar{\mu} m_{\mathfrak{p}}\right)^{1 / 2}, \gamma=5 / 3$, and $\lambda=0.25$ are adopted (e.g., Allen et al. 2006). For the gas rotating with a small angular velocity $\Omega_{\mathrm{B}}\left(\Omega_{\mathrm{B}} \ll \Omega_{\mathrm{K}}\right.$, and $\Omega_{\mathrm{K}}$ is the Keplerian velocity) at the Bondi radius, the gas falls almost freely to the black hole without loosing its angular momentum till the circularization radius. An accretion disk is formed within the circularization radius $R_{\mathrm{c}}$, which means that it roughly corresponds to the outer radius of the disk, i.e., $R_{\text {out }} \simeq R_{\mathrm{c}}$. At the circularization radius $R_{\mathrm{c}}$, the gas is rotating at the Keplerian velocity. This leads to

$$
R_{\mathrm{B}}^{2} \Omega_{\mathrm{B}}=R_{\mathrm{c}}^{2} \Omega_{\mathrm{K}}\left(R_{\mathrm{c}}\right)
$$

i.e.,

$$
R_{\mathrm{c}}=R_{\mathrm{B}} \tilde{\Omega}_{\mathrm{B}}^{2}
$$

where $\tilde{\Omega}_{\mathrm{B}}=\Omega_{\mathrm{B}} / \Omega_{\mathrm{K}}\left(R_{\mathrm{B}}\right)$. Suppose a weak vertical magnetic field $B_{\text {ext }}$ threading the gas at the Bondi radius, we can estimate the field strength at the circularization radius as

$$
B\left(R_{\mathrm{c}}\right) \simeq\left(\frac{R_{\mathrm{B}}}{R_{\mathrm{c}}}\right)^{2} B_{\mathrm{ext}} .
$$

\subsection{Advection of the magnetic field in the thin accretion disk}

As the advection of the field in a thin accretion disk is quite inefficient, we consider the field advection in the disk predominantly driven by the magnetic outflows in this section (see Cao \& Spruit 2013, for the details). If the magnetic field is sufficiently strong in the disk, a fraction of the gas at the disk surface (or the hot corona above the disk) may be accelerated into the outflows by the field lines threading the rotating disk. Such outflows may carry a large amount of the angular momentum of the gas in the disk, which may alter the disk structure substantially (Cao \& Spruit 2013; Li 2014; Cao 2016, Cao \& Lai in preparation). The radial velocity of the disk is significantly increased due to the presence of the outflows. In this case, the radial velocity of the disk with magnetic outflows is

$$
V_{R} \simeq V_{R, \mathrm{vis}}+V_{R, \mathrm{~m}}
$$

where the first term is due to the conventional turbulence in the disk, $V_{R}=-\alpha c_{\mathrm{S}} H / R$, and the second term is contributed by the outflows,

$$
V_{R, \mathrm{~m}}=-\frac{2 T_{\mathrm{m}}}{R \Sigma \Omega}
$$

For an accretion disk with magnetically driven outflows, the magnetic torque exerted by the outflows in unit area of the disk surface is

$$
T_{\mathrm{m}}=\frac{B_{z} B_{\phi}^{\mathrm{s}}}{2 \pi} R=\frac{\xi_{\phi} B_{z}^{2}}{2 \pi} R,
$$

where $B_{\phi}^{\mathrm{s}}$ is the azimuthal component of the large scale magnetic field at the disk surface, and $\xi_{\phi}=B_{\phi}^{\mathrm{s}} / B_{z}$. We use a parameter $f_{\mathrm{m}}\left(f_{\mathrm{m}}=V_{R, \mathrm{~m}} / V_{R \text {,vis }}\right)$ to describe the relative importance of the outflows on the radial velocity of the disk,

$$
\begin{aligned}
V_{R}= & \left(1+f_{\mathrm{m}}\right) V_{R, \mathrm{vis}}=\left(1+\frac{1}{f_{\mathrm{m}}}\right) V_{R, \mathrm{~m}} \\
& =-\left(1+\frac{1}{f_{\mathrm{m}}}\right) \frac{2 T_{\mathrm{m}}}{R \Sigma \Omega}=-\left(1+\frac{1}{f_{\mathrm{m}}}\right) \frac{B_{z} B_{\phi}^{\mathrm{s}}}{\pi \Sigma \Omega} .
\end{aligned}
$$


The value of $f_{\mathrm{m}}$ is predominantly determined by the properties of the magnetically driven outflows.

The mass accretion rate of the disk at the outer radius $\left(R_{\text {out }}=\right.$ $\left.R_{\mathrm{c}}\right)$ is

$$
\dot{M}=-2 \pi R_{\text {out }} \Sigma\left(R_{\text {out }}\right) V_{R}\left(R_{\text {out }}\right)=2\left(1+\frac{1}{f_{\mathrm{m}}}\right) \xi_{\phi} R_{\text {out }} B_{z}^{2} \Omega^{-1} .
$$

Substituting Equations (2), (4) and (5) into Equation (10), we obtain

$$
\begin{aligned}
\tilde{\Omega}_{\mathrm{B}} & =0.336 \xi_{\phi}^{2 / 3}\left(1+\frac{1}{f_{\mathrm{m}}}\right)^{1 / 3} \\
& \times\left(\frac{T_{\mathrm{B}}}{\mathrm{keV}}\right)^{-5 / 6}\left(\frac{B_{\text {ext }}}{\text { mGauss }}\right)^{2 / 3}\left(\frac{M_{\mathrm{bh}}}{10^{8} M_{\odot}}\right)^{1 / 3} \dot{m}^{-1 / 3},
\end{aligned}
$$

where $T_{\mathrm{B}}$ is the gas temperature at $R_{\mathrm{B}}$, and the dimensionless mass accretion rate $\dot{m}$ is defined as

$$
\dot{m}=\frac{\dot{M}}{\dot{M}_{\text {Edd }}}, \quad \dot{M}_{\text {Edd }}=1.39 \times 10^{18}\left(\frac{M_{\mathrm{bh}}}{M_{\odot}}\right) \mathrm{g} \mathrm{s}^{-1} .
$$

As the ratio $\xi_{\phi}$ is in general required to be $\lesssim 1$ (see the detailed discussion in Livio et al. 1999), we derive the first condition for efficient field advection in an accretion disk with magnetic outflows as

$$
\begin{aligned}
& \tilde{\Omega}_{\mathrm{B}}<0.336\left(1+\frac{1}{f_{\mathrm{m}}}\right)^{1 / 3} \\
& \times\left(\frac{T_{\mathrm{B}}}{\mathrm{keV}}\right)^{-5 / 6}\left(\frac{B_{\text {ext }}}{\mathrm{mGauss}}\right)^{2 / 3}\left(\frac{M_{\mathrm{bh}}}{10^{8} M_{\odot}}\right)^{1 / 3} \dot{m}^{-1 / 3},
\end{aligned}
$$

The radial advection timescale of the magnetic field in the disk is

$$
\tau_{\mathrm{adv}} \sim \frac{R}{\left|V_{R}\right|}
$$

The magnetic diffusion timescale is

$$
\tau_{\text {dif }} \sim \frac{R H \kappa_{0}}{\eta},
$$

where $\kappa_{0}=B_{z} / B_{r}^{\mathrm{s}}$ at the disk surface, and $\eta$ is the magnetic diffusivity. For a steady magnetic field, the inclination of the field line at the disk surface can be estimated by equating the radial advection timescale with the magnetic diffusion timescale, i.e., $\tau_{\text {adv }}=\tau_{\text {dif }}$ (Lubow et al. 1994). This leads to

$$
\left|V_{R}\right|=\frac{\alpha P_{\mathrm{m}} c_{\mathrm{s}}}{\kappa_{0}},
$$

where the magnetic Prandtl number $P_{\mathrm{m}}=\eta / \nu$, and the viscosity $\nu=\alpha c_{\mathrm{s}} H$. Comparing Equation (9) with Equation (16), we have

$$
\kappa_{0}=\frac{1}{1+f_{\mathrm{m}}} \frac{P_{\mathrm{m}}}{\tilde{H}}
$$

where the relative disk thickness $\tilde{H}=H / R$. The cold gas can be accelerated by the magnetic field from the midplane of a Keplerian disk if the field line is inclined at $\lesssim 60^{\circ}\left(\kappa_{0} \lesssim \sqrt{3}\right)$ with respect to the disk surface (Blandford \& Payne 1982). The inclination angle could be a bit higher than $60^{\circ}$ for the hot gas accelerated by the magnetic field with the help of the thermal pressure or/and the radiation force of the disk (Cao \& Spruit 1994; Cao 2012, 2014). For a disk without magnetic outflows, $\kappa_{0}=P_{\mathrm{m}} / \tilde{H}=\tilde{H}^{-1}$ if $P_{\mathrm{m}}=1$. It means that the gas cannot be magnetically accelerated by the magnetic field formed in a thin, viscously driven accretion disk, because $\kappa_{0} \sim P_{\mathrm{m}} / \tilde{H} \gg 1$ for a thin disk, i.e., the field lines are nearly vertically threading the disk. For a thin accretion disk predominantly driven by the outflows with $\tilde{H}=0.1, \kappa_{0} \simeq 1.7$ if $f_{\mathrm{m}}=5$ for a typical value of $P_{\mathrm{m}}=1$. This indicates that a strong magnetic field can be formed in a thin disk if most of its angular momentum is removed by the outflows.

The gravitational energy dissipation rate from the unit area of the disk is

$$
Q_{+}=\frac{1}{2} \nu \Sigma\left(R \frac{d \Omega}{d R}\right)^{2},
$$

and the mass accretion rate is

$$
\dot{M}=-2 \pi R \Sigma V_{R}=-4 \pi R\left(R \frac{d \Omega}{d R}\right)^{-2} \nu^{-1} V_{R, \mathrm{vis}}\left(1+f_{\mathrm{m}}\right) Q_{+},
$$

where Equation (9) is used. For a fixed $Q_{+}$(disk luminosity), the mass accretion rate of an accretion disk with magnetic outflows is $f_{\mathrm{m}}$ times higher than that of a conventional viscously driven accretion disk, because most of the gravitational power of the gas in the disk is tapped into the outflows by the magnetic field.

In the outer region of the disk, the local gravity of the disk may dominate over the gravity of the black hole. In the region beyond a critical radius $R_{\mathrm{T}}$, it may become a clumpy disk due to the gravitational instability (Hure et al. 1994; Collin \& Huré 2001). The critical radius $R_{\mathrm{T}}$ can be estimated with the Toomre parameter (Toomre 1964; Goldreich \& Lynden-Bell 1965),

$$
Q_{\mathrm{T}}=\frac{\Omega_{\mathrm{K}} c_{\mathrm{s}, \mathrm{d}}}{2 \pi G \rho_{\mathrm{d}} H}=1
$$

The local structure of a disk accreting at a mass rate $\dot{M}$ with magnetically driven outflows, is the same as a standard thin disk accreting at $\dot{M} /\left(1+f_{\mathrm{m}}\right)$. The critical radius $R_{\mathrm{T}}$ can be calculated with the standard accretion disk model when the values of the parameter $f_{\mathrm{m}}$ and other disk parameters are specified.

The accretion of the clumpy disk may be driven by the collisions of the clumpies, which is similar to the case of dust torus in AGNs (Krolik \& Begelman 1988). The large scale magnetic field threading the clumps may not be able to survive through repeatedly collisions. A clumpy disk region would be an obstacle for accumulation of external magnetic field. To avoid such a clumpy region in the disk, one requires $R_{\mathrm{c}}<R_{\mathrm{T}}$, i.e.,

$$
\tilde{\Omega}_{\mathrm{B}} \leq\left(\frac{R_{\mathrm{T}}}{R_{\mathrm{B}}}\right)^{1 / 2}=0.0017 r_{\mathrm{T}}^{1 / 2}\left(\frac{k T_{\mathrm{B}}}{1 \mathrm{keV}}\right)^{1 / 2},
$$

where

$$
r_{\mathrm{T}}=\frac{R_{\mathrm{T}}}{R_{\mathrm{S}}}, \quad R_{\mathrm{S}}=\frac{2 G M_{\mathrm{bh}}}{c^{2}},
$$

and Equation (1) is used. This is the second condition for efficient field advection in an accretion disk with magnetic outflows. 


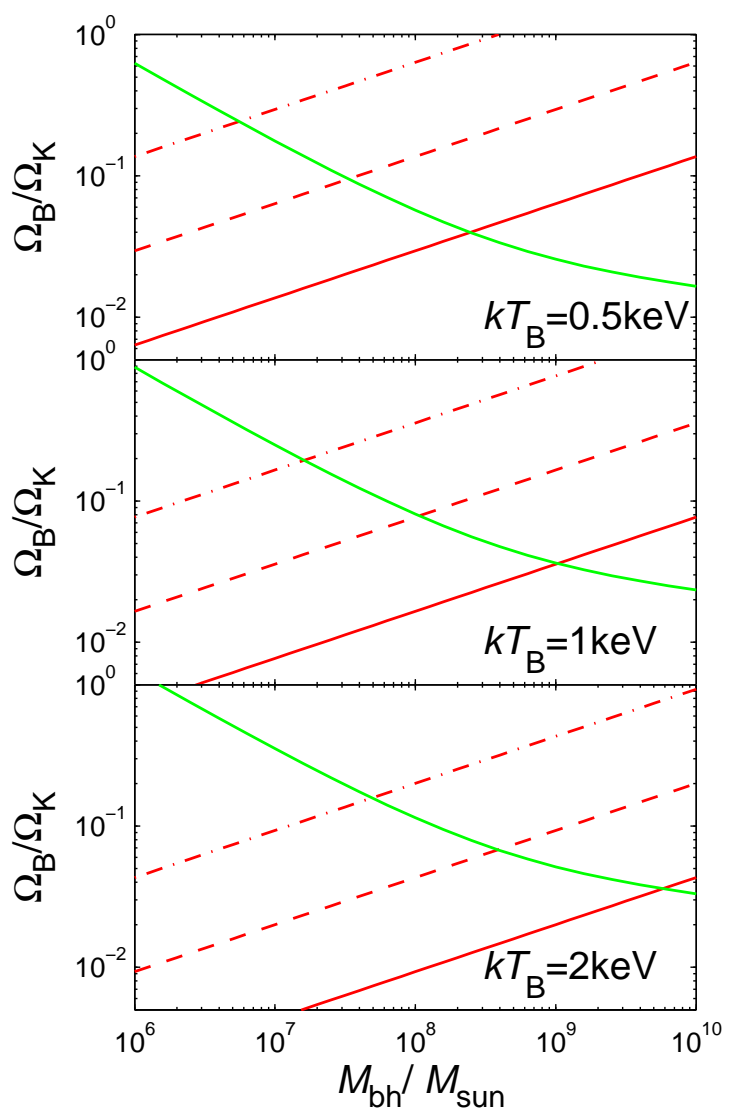

FIG. 1. - The critical angular velocities of the gas at the Bondi radius as functions of the black hole mass. The external field can be efficiently dragged in by an accretion disk with magnetic outflows only if the angular velocity of the gas is lower than both of the two critical values. All the calculations are carried out with $\dot{m}=1$. The red lines correspond to the first condition (see Equation 13, and the green lines to the second condition for efficient field advection in the accretion disk with magnetic outflows (see Equation 21. The viscosity parameter $\alpha=1$ is adopted in the calculations. The different types of the red lines indicate the results with different values of the external field strength at the Bondi radius (solid: $0.01 \mathrm{mGauss,}$ dashed: $0.1 \mathrm{mGauss}$, and dash-dotted: 1 mGauss).

\section{RESULTS}

In Section 2.2, two constraints on the angular velocity of the gas at the Bondi radius are derived. The external field can be efficiently advected in an accretion disk with magnetic outflows, only if the gas with angular velocity lower than both of these two critical values at the Bondi radius. In Figure1, we plot the results varying with the black hole mass for different gas temperatures and external field strengths. It is found that the angular velocity $\Omega_{\mathrm{B}}$ is always required to be much lower than the Keplerian velocity, in order to form a strong field in the inner region of the disk, though the detailed results also vary with the other parameters. The results varying with the mass accretion rate are plotted in Figure 2. As discussed in Section 2.2, the model parameter $f_{\mathrm{m}}=5$ is adopted in all the calculations.

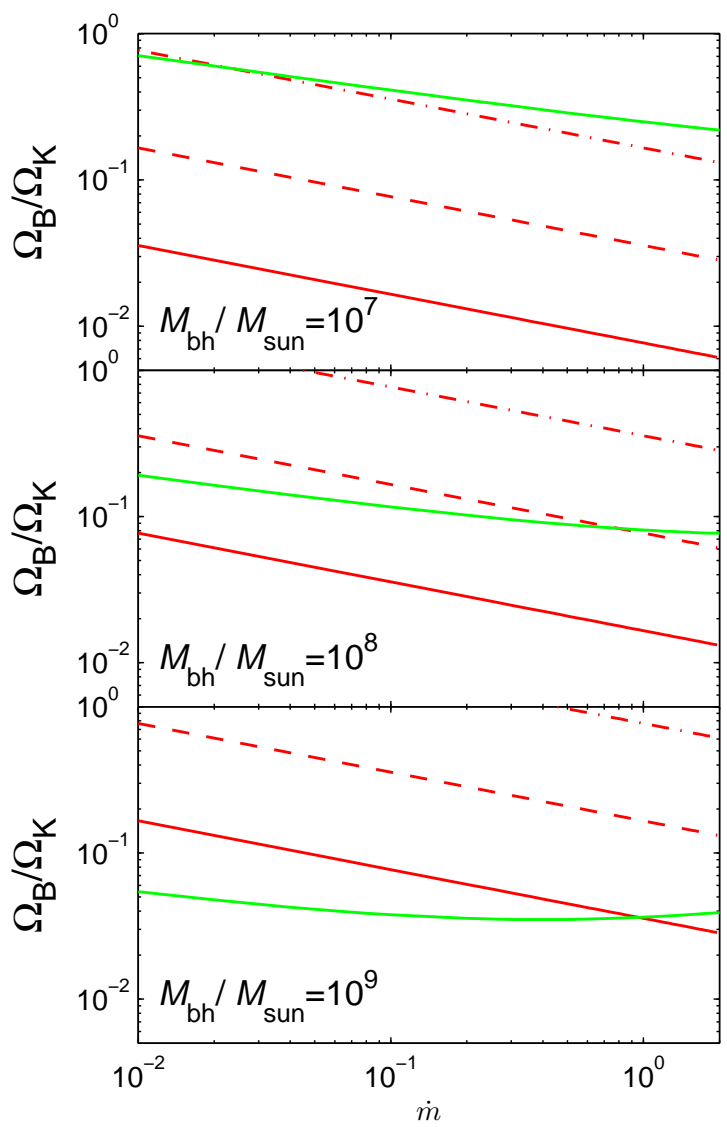

FIG. 2.- Same as Figure 1 but for the results as functions of the mass accretion rate at the Bondi radius. The circumnuclear gas temperature $k T_{\mathrm{B}}=$ $1 \mathrm{keV}$ is adopted in the calculations.

\section{DISCUSSION}

There are two conditions that should be satisfied for efficient advection of the field in the thin disk with magnetic outflows. The first condition is a sufficiently strong magnetic field at the outer radius (circularization radius) of the disk (Equation 13), and the second one is the disk not being suffered from the gravitational instability, i.e., the local gravity of the disk should not dominate over the gravity of the black hole (Equation 21).

We find that RL AGNs are mainly constrained by the second condition for the AGNs with most massive black holes ( $\gtrsim 10^{9} M_{\odot}$ ). For the moderate or small black holes, RL AGNs are predominantly controlled by the first condition, unless for a strong external magnetic field with $B_{\text {ext }} \gtrsim 0.1$ mGauss is present (see Figure 2). In this case, the critical angular velocity of the gas at the Bondi radius, below which an AGN may appears as a RL AGN, increases with increasing black hole mass if all the other parameters are fixed.

Typical magnetic field strengths of galaxy cluster atmospheres are at the order of $\sim \mu$ Gauss (see Carilli \& Taylor 2002, and the references therein), and the field strengths could be stronger for the ISM in galaxies (Thompson et al. 2006; de Gasperin et al. 2012). In the central region of our galaxy, the field strength of the gas can be as high as $\sim$ mGauss (Han \& Zhang 2007). A weaker magnetic field requires the gas with a lower angular momentum at the Bondi radius for RL AGNs, because the circularization radius decreases with 
decreasing angular momentum of the gas, which increases the amplification of the external magnetic field in the region between the Bondi radius and the circularization radius. Using Chandra X-ray observations of nine nearby radio galaxies, Allen et al. (2006) measured the temperatures of the gas at the Bondi radius of these galaxies, which are in the range of $\sim 0.5-1.3 \mathrm{keV}$. We find that our main results are insensitive to the gas temperature (see Figure 1).

The radio properties of AGNs are found to be linked to their host galaxies (e.g., de Ruiter et al. 2005; Balmaverde \& Capetti 2006; Capetti \& Balmaverde 2006). Capetti \& Balmaverde (2005) compiled a sample of AGNs in nearby early-type galaxies with available archival HST images. The sources are classified with HST images into "core" and "power-law" galaxies, and they found that the core galaxies invariably host a radio-loud nucleus Balmaverde \& Capetti 2006; Capetti \& Balmaverde 2006). The core galaxies are slowly rotating and have boxy isophotes, while the power-law galaxies rotate rapidly and are disky (Capetti \& Balmaverde 2005). This supports the model suggested in this paper that the source accreting the gas with a low angular velocity may preferentially appear as an RL AGN.

The mass accretion rate is about $f_{\mathrm{m}}$ times higher than a conventional accretion disk with the same luminosity (see Equation [19), which implies that the mass growth of the black holes in RL AGNs is much faster than that in RQ AGNs with the same luminosity. This is consistent with the fact that the massive black holes in RL quasars are systematically a few times heavier than those in their RQ counterparts (Laor 2000; McLure \& Jarvis 2004). As the black hole acquires the angular momentum of the gas in the disk, the hole is spun up through accretion simultaneously. It may imply that most massive black holes in RL AGNs are rotating rapidly, and the BZ mechanism may also play an important role in RL AGNs.

Due to the mass loss in the outflows, the mass accretion rate decreases with decreasing radius in the disk. The mass loss rate in the outflows is governed by the magnetic field configuration/strength and the disk properties (e.g., disk temperature and density). The present analysis focuses on the necessary conditions for an accretion disk-outflow system, and the minimal field strength at the outer radius of the disk is derived for launching strong outflows. The properties of the outflow can be derived with the magnetic outflow solution, if suitable boundary conditions are provided (e.g., Cao \& Spruit 1994; $\mathrm{CaO}$ 2014), which is beyond the scope of this work.

Our model implies that RL AGNs are closely associated with the magnetic outflows. The blueshifted Fe K absorption lines in the $\mathrm{X}$-ray spectra of broad-line radio galaxies have been observed with Suzaku, which indicates the ultrafast outflows co-exist with the relativistic jets in these sources (Tombesi et al. 2010). This seems to be consistent with our model.

Advection dominated accretion flows (ADAFs) may probably surround the black holes in low-luminosity AGNs
(Narayan \& Yi 1994, 1995, see Yuan \& Narayan 2014 for a recent review). Although it has already been shown that the external magnetic field can be efficiently advected in an ADAF ( $\mathrm{CaO}$ 2011), the field enhancement in the region from the Bondi radius to the circularization radius provides a stronger field to be dragged in by the ADAF. Thus, the circumnuclear gas with a smaller angular velocity may help to form a stronger field in the inner region of the ADAF in lowluminosity AGNs.

The putative dust torus is an important ingredient of the unification model for AGN (Antonucci 1993). It is known that the mid-IR spectra and IR-to-optical flux ratios are very similar in RL and RQ quasars (e.g., Shang et al. 2011; Gupta et al. 2016), and dust torus may also be present in most RL quasars if not all. We conjecture that, the hot gas feeding the black hole co-exists with the dust clumps in a region beyond the circularization radius, and the dynamics of the hot gas is not altered significantly by the motion of the dust clumps in the torus. If this is the case, the accretion of the hot gas from the Bondi radius to the black hole can still be well described by the calculations in this paper even if a putative dust torus is present.

\section{SUMMARY}

The circumnuclear gas with a low angular velocity falls nearly freely from the Bondi radius to form an accretion disk within the circularization radius. The external magnetic field threading the gas is strongly amplified in this region due to the field flux freezing. For the gas with an angular velocity lower than a critical value, the field in the disk is strong enough to drive magnetic outflows, which carries away most of the angular momentum of the disk. This strongly increases the radial velocity of the disk, and therefore the field can be efficiently dragged inwards by the disk. Relativistic jets may be driven by such a large scale magnetic field through either the BP or BZ mechanism. In this case, the object may appear as an RL AGN.

If the angular velocity of the circumnuclei gas is larger than a critical value, the circularization radius (i.e., the outer radius of the disk) becomes larger, and the field cannot be amplified to a strong field in the disk to accelerate outflows. Thus, a conventional turbulence driven thin disk is formed within the circularization radius, and the advection of the field in the disk is rather inefficient. In this case, no relativistic jet is formed in the inner region of the disk, which corresponds to an RL AGN.

I thank the referee for the helpful comments/sugestions. This work is supported by the NSFC (grant 11233006), the Strategic Priority Research Program ąrthe Emergence of Cosmological Structuresąs of the CAS (grant No. XDB09000000), the CAS/SAFEA International Partnership Program for Creative Research Teams, and Shanghai Municipality.

\section{REFERENCES}

\author{
Allen, S. W., Dunn, R. J. H., Fabian, A. C., Taylor, G. B., \& Reynolds, C. S. \\ 2006, MNRAS, 372, 21 \\ Antonucci, R. 1993, ARA\&A, 31, 473 \\ Balmaverde, B., \& Capetti, A. 2006, A\&A, 447, 97 \\ Baloković, M., Smolčić, V., Ivezić, Ž., et al. 2012, ApJ, 759, 30 \\ Bisnovatyi-Kogan, G. S., \& Ruzmaikin, A. A. 1974, Ap\&SS, 28, 45
}

Bisnovatyi-Kogan, G. S., \& Ruzmaikin, A. A. 1976, Ap\&SS, 42, 401

Blandford, R. D., \& Payne, D. G. 1982, MNRAS, 199, 883

Blandford, R. D., \& Znajek, R. L. 1977, MNRAS, 179, 433

Bu, D.-F., \& Yuan, F. 2014, MNRAS, 442, 917

Cao, X. 2002, MNRAS, 332, 999

Cao, X. 2003, ApJ, 599, 147 
Cao, X. 2011, ApJ, 737, 94

Cao, X. 2012, MNRAS, 426, 2813

Cao, X. 2014, ApJ, 783, 51

Cao, X. 2016, ApJ, 817, 71

Cao, X., \& Lai, D., in preparation

Cao, X., \& Spruit, H. C. 1994, A\&A, 287, 80

Cao, X., \& Spruit, H. C. 2013, ApJ, 765, 149

Capetti, A., \& Balmaverde, B. 2005, A\&A, 440, 73

Capetti, A., \& Balmaverde, B. 2006, A\&A, 453, 27

Carilli, C. L., \& Taylor, G. B. 2002, ARA\&A, 40, 319

Cirasuolo, M., Magliocchetti, M., Celotti, A., \& Danese, L. 2003, MNRAS, 341,993

Collin, S., \& Huré, J.-M. 2001, A\&A, 372, 50

de Gasperin, F., Orrú, E., Murgia, M., et al. 2012, A\&A, 547, A56

de Ruiter, H. R., Parma, P., Capetti, A., et al. 2005, A\&A, 439, 487

Fender, R. P., Gallo, E., \& Russell, D. 2010, MNRAS, 406, 1425

Fromang, S., \& Stone, J. M. 2009, A\&A, 507, 19

Goldreich, P., \& Lynden-Bell, D. 1965, MNRAS, 130, 97

Guan, X., \& Gammie, C. F. 2009, ApJ, 697, 1901

Guilet, J., \& Ogilvie, G. I. 2012, MNRAS, 424, 2097

Guilet, J., \& Ogilvie, G. I. 2013, MNRAS, 430, 822

Gupta, M., Sikora, M., \& Nalewajko, K. 2016, MNRAS, 461, 2346

Han, J. L., \& Zhang, J. S. 2007, A\&A, 464, 609

Hure, J.-M., Collin-Souffrin, S., Le Bourlot, J., \& Pineau des Forets, G. 1994, A\&A, 290, 19

Kellermann, K. I., Sramek, R., Schmidt, M., Shaffer, D. B., \& Green, R. 1989, AJ, 98, 1195

King, A. R., Pringle, J. E., \& Hofmann, J. A. 2008, MNRAS, 385, 1621

Krolik, J. H., \& Begelman, M. C. 1988, ApJ, 329, 702

Laor, A. 2000, ApJ, 543, L111

Lesur, G., \& Longaretti, P.-Y. 2009, A\&A, 504, 309

Li, S.-L. 2014, ApJ, 788, 71

Li, Y.-R., Wang, J.-M., \& Ho, L. C. 2012, ApJ, 749, 187

Livio, M., Ogilvie, G. I., \& Pringle, J. E. 1999, ApJ, 512, 100
Lovelace, R. V. E., Rothstein, D. M., \& Bisnovatyi-Kogan, G. S. 2009, ApJ, 701,885

Lubow, S. H., Papaloizou, J. C. B., \& Pringle, J. E. 1994, MNRAS, 267, 235

McLure, R. J., \& Jarvis, M. J. 2004, MNRAS, 353, L45

Melia, F., Liu, S., \& Coker, R. 2001, ApJ, 553, 146

Narayan, R., \& McClintock, J. E. 2012, MNRAS, 419, L69

Narayan, R., \& Yi, I. 1994, ApJ, 428, L13

Narayan, R., \& Yi, I. 1995, ApJ, 452, 710

Ogilvie, G. I., \& Livio, M. 2001, ApJ, 553, 158

Parker, E. N. 1979, in Chapter 17, Cosmical Magnetic Fields (Oxford:Clarendon Press)

Shang, Z., Brotherton, M. S., Wills, B. J., et al. 2011, ApJS, 196, 2

Sikora, M., \& Begelman, M. C. 2013, ApJ, 764, L24

Sikora, M., Begelman, M. C., Madejski, G. M., \& Lasota, J.-P. 2005, ApJ, 625,72

Sikora, M., Stawarz, Ł., \& Lasota, J.-P. 2007, ApJ, 658, 815

Sikora, M., Stawarz, Ł., \& Lasota, J.-P. 2008, New A Rev., 51, 891

Spruit, H. C., \& Uzdensky, D. A. 2005, ApJ, 629, 960

Tchekhovskoy, A., Narayan, R., \& McKinney, J. C. 2010, ApJ, 711, 50

Tchekhovskoy, A., Narayan, R., \& McKinney, J. C. 2011, MNRAS, 418, L79

Thompson, T. A., Quataert, E., Waxman, E., Murray, N., \& Martin, C. L. 2006, ApJ, 645, 186

Tombesi, F., Sambruna, R. M., Reeves, J. N., et al. 2010, ApJ, 719, 700

Toomre, A. 1964, ApJ, 139, 1217

van Ballegooijen, A. A. 1989, in Accretion Disks and Magnetic Fields in Astrophysics, ed. G. Belvedere (ASSL Vol. 156; Dordrecht: Kluwer), 99 Volonteri, M., Sikora, M., \& Lasota, J.-P. 2007, ApJ, 667, 704

Volonteri, M., Sikora, M., Lasota, J.-P., \& Merloni, A. 2013, ApJ, 775, 94

Wilson, A. S., \& Colbert, E. J. M. 1995, ApJ, 438, 62

Xu, C., Livio, M., \& Baum, S. 1999, AJ, 118, 1169

Ye, Y.-C., \& Wang, D.-X. 2005, MNRAS, 357, 1155

Yousef, T. A., Brandenburg, A., \& Rüdiger, G. 2003, A\&A, 411, 321

Yuan, F., \& Narayan, R. 2014, ARA\&A, 52, 529 